\title{
Avoiding the Obstacles in the Robot Working Zone by using the Lee Algorithm
}

\author{
Naqib Daneshjo', Marián Kralík², Katka Petrovčiková1, \\ Erika Dudáš Pajerská', Michael Paštéka² \\ 1 Faculty of Business Economics with seat in Kosice, University of Economics in Bratislava, Slovakia \\ 2 Slovak University of Technology in Bratislava, Faculty of Mechanical Engineering, Namestie Slobody 17, \\ 81231 Bratislava, Slovakia \\ * Corresponding author's e-mail: daneshjo47@gmail.com
}

\begin{abstract}
Non-collision trajectories of an industrial robot are the key objective when planning its handling cycles. When the robot should move optimally, precisely and safely in each situation, it is necessary that every obstacle placed in its transition trajectory should be programmed and avoided. The optimal trajectory corresponds to choosing, if possible, the shortest and most appropriate way for the specific movement to be finished. The task of avoiding obstacles by the industrial robot in its working zone can be solved by various methods and algorithms. The paper shows the use of the kinematics SCARA as a way of avoiding obstacles by a robot in the working zone using the Lee algorithm. The configuration fields and networks are being modelled for the avoidance of obstacles together with their evaluation and the search for the shortest trajectory reflected in the robot programming.
\end{abstract}

Keywords: robot working zone, Lee algorithm, local coordinates system, general coordinates system

\section{INTRODUCTION}

There are a few ways of describing the relative position of the individual elements of the kinematic robot mechanism. The grip angle elements can be used as the revolute pair; on the other hand, the movable kinematic pair involved the distance from the middle of the moving unit from the unit edge used for the movement $[1,2]$. From the practical point of view, in kinematic mechanisms with the moving elements, ir is most practical to describe their mutual position using the system of the so-called local coordinates system (LCS) which are firmly connected (they are moving mutually with the elements) and the current position and orientation toward the general coordinates system (GCS) of which can be easily deducted.

\section{THE LEE ALGORITHM APPLICATION TO AVOID OBSTACLES IN THE ROBOT WORKING ZONE}

The Lee algorithm can be used for the labyrinth problem solving in directing individual robot trajectories. Its task is to identify the shortest trajectory between two points in the "labyrinth". The main problems involve creation of a configuration field in which the obstacle occurs, its evaluation and the following search for the optimal trajectory [7]. The configuration field is the collection of all the positions that can be reached by the robot caudal effector. They are expressed in the articulated coordinates $[3,4,9]$.

The configuration is the sequenced location coordinates n-tuple $\left(\mathrm{q}_{1}-\mathrm{q}_{\mathrm{n}}\right)$ by which the mutual position and the orientation of all the arm elements by the effector is specifically described. 
The objective of the Lee algorithm application is to find the non-collision path of the robot around the obstacle. The application can be divided into the following steps:

1. Definition of the obstacle that needs to be avoided.

2. Configuration field creation.

3. Evaluation the configuration field.

4. Optimal trajectory identification.

The obstacle that the robot with SCARA kinematics has to avoid by its motion is the cuboid $(\mathrm{H} \times \mathrm{W} \times \mathrm{L}=117 \times 59 \times 166 \mathrm{~mm})$; its model was created using the CATIA software $[6,8]$.

For the configuration field creation, it is necessary to display this obstacle in the xy plane. The configuration field is created as the dependence upon the rotation (parameter) $\mathrm{q}_{2}$ from the rotation (parameter) $q_{1}$, the values of the both rotations are necessary for its creation $[5,10]$. The $\mathrm{q}_{1}$ and $\mathrm{q}_{2}$ values can be acquired by using the

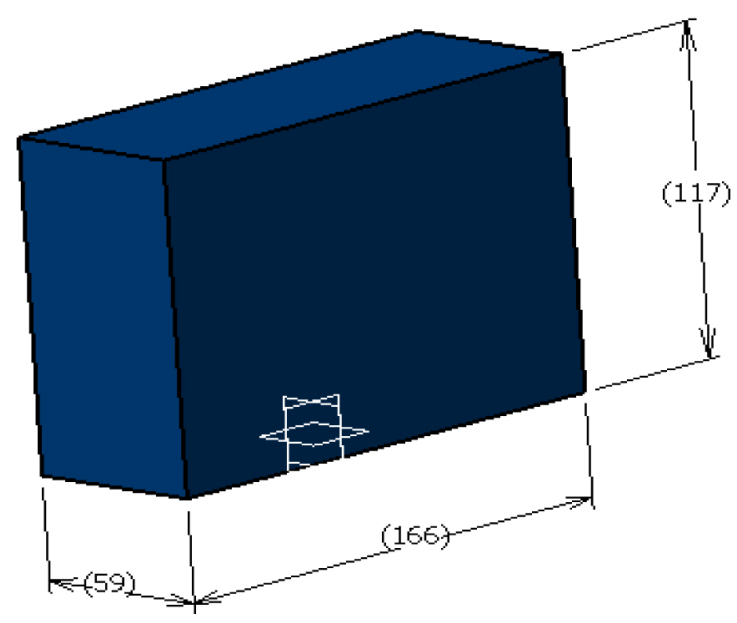

Figure 1. The obstacle model final formulas of the two pairs of the inverse task solution, see Figure 2.

$$
\begin{gathered}
\boldsymbol{q}_{\mathbf{2}}^{\prime}=\arccos \left[\frac{-a_{1}^{2}-a_{2}^{2}+x_{E}^{2}+y_{E}^{2}}{2 a_{1} a_{2}}\right] \\
\boldsymbol{q}_{\mathbf{1}}^{\prime}=\operatorname{arctg} \frac{y_{E}}{x_{E}}-\arccos \mid \\
{\left[\frac{x_{E}^{2}+y_{E}^{2}+a_{1}^{2}-a_{2}^{2}}{2 a_{1} \sqrt{x_{E}^{2}+y_{E}^{2}}}\right]}
\end{gathered}
$$

While calculating the $\beta$ and $\varphi$ angles the value of $\mathrm{q}_{1}$ parameter can be determined:

$$
\begin{aligned}
& \operatorname{arctg} \frac{y_{E}}{x_{E}}+\arccos \\
& {\left[\frac{x_{E}^{2}+y_{E}^{2}+a_{1}^{2}-a_{2}^{2}}{2 a_{1} \sqrt{x_{E}^{2}+y_{E}^{2}}}\right]}
\end{aligned}
$$

The $\mathrm{q}_{2}$ parameter is also calculated using the law of cosine:

$$
\boldsymbol{q}_{2}=-\arccos \left[\frac{-a_{1}^{2}-a_{2}^{2}+x_{E}^{2}+y_{E}^{2}}{2 a_{1} a_{2}}\right]
$$

These are the pairs of formulas: the first are the solutions (1) and (2) and second are the solutions (3) and (4). The input data for the formulas is the values of the $x, y$ axes of a defined obstacle. Therefore, the obstacle, more precisely its image in the xy plane is divided into the individual points, where their mutual distances in the direction of the axis $\mathrm{x}$ and $\mathrm{y}$ are equal to $5 \mathrm{~mm}$ (see Table 1). From the point of view of avoiding the obstacle, it is also important to take into consideration the

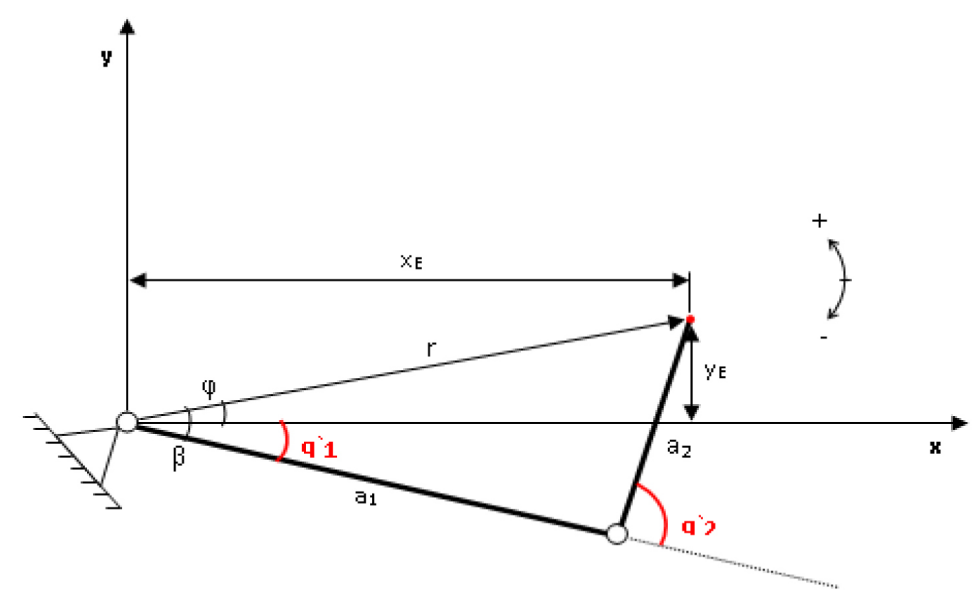

Figure 2. Robot arm display with SCARA Kinematics in the xy field for the calculation of $\mathrm{q}_{1}$ and $\mathrm{q}_{2}$ parameters 
size of the end robot effector; in this case - the draught tube $\varnothing 23 \mathrm{~mm}$. Table 1 also contains the points coordinates (the mutual distances in the direction of axis $\mathrm{x}$ and $\mathrm{y}$ equal to $5 \mathrm{~mm}$ as well) the so-called "protection zone" which is around each of the obstacles in the distance of $14 \mathrm{~mm}$ from each of its sides. Together with the obstacle coordinates and its protection zone, Table 1 defines the position of the starting and the end point of the robot trajectory in the xy plane.

\section{Configuration field creation - network creation in which the obstacle is placed}

The configuration field creation denotes the network creation in which the obstacle is placed. The configuration field itself is created as the dependence of the rotation (parameter) $q_{2}$ to the rotation (parameter) $\mathrm{q}_{1}$. Thus, the main task is to calculate the values of both rotations for the points presenting the obstacle and its protection zone, so that its position in the configuration field is clearly defined. The values for $\mathrm{q}_{1}$ a $\mathrm{q}_{2}$ are acquired using the final formulas of the two pairs of inverse task solution. These are pairs of formulas - the first are the formulas (1) and (2) and the second are the formulas (3) and (4). The input data are $\mathrm{x}$ and $\mathrm{y}$ coordinates from Table 1 and the values $\mathrm{a}_{1}$ and $\mathrm{a}_{2}$ are the lengths of the robot's arms $\left(a_{1}=225 \mathrm{~mm}, \mathrm{a}_{2}=166.1 \mathrm{~mm}\right)$. The calculation results are the two solutions of the obstacle placement and its protection zone in the configuration field (see Table 3).

For both solutions it is necessary to construct, within the configuration point, the network of those configurations. Each solution has its own configuration field due to the choice of different increases in the direction of the axis $\mathrm{q}_{1}$ and $\mathrm{q}_{2}$ (increases are shown in the Table 2).

Defining the distances of the individual axes in the network is important for the precise determination of the $\mathrm{q}_{1}$ and $\mathrm{q}_{2}$ rotation values of individual configurations (network points) because after identifying the optimal trajectory, these values

Table 1. Coordinates for the obstacle points, protection zone, starting and end trajectory points

\begin{tabular}{|c|c|c|c|c|c|}
\hline \multicolumn{3}{|c|}{ Obstacle } & \multicolumn{3}{|c|}{ Protection zone } \\
\hline & $\mathrm{x}_{\mathrm{E}}[\mathrm{mm}]$ & $\mathrm{y}_{\mathrm{E}}[\mathrm{mm}]$ & & $\mathrm{x}_{\mathrm{E}}[\mathrm{mm}]$ & $\mathrm{y}_{\mathrm{E}}[\mathrm{mm}]$ \\
\hline point $A$ & 190.63 & -188.87 & point $A_{2}$ & 176.63 & -202.87 \\
\hline \multirow{11}{*}{ side $A B$} & 195.63 & -188.87 & \multirow{14}{*}{ side $A_{2} B_{2}$} & 190.63 & -202.87 \\
\hline & 200.63 & -188.87 & & 190.63 & -202.87 \\
\hline & 205.63 & -188.87 & & 195.63 & -202.87 \\
\hline & 210.63 & -188.87 & & 200.63 & -202.87 \\
\hline & 215.63 & -188.87 & & 205.63 & -202.87 \\
\hline & 220.63 & -188.87 & & 210.63 & -202.87 \\
\hline & 225.63 & -188.87 & & 215.63 & -202.87 \\
\hline & 230.63 & -188.87 & & 220.63 & -202.87 \\
\hline & 235.63 & -188.87 & & 225.63 & -202.87 \\
\hline & 240.63 & -188.87 & & 230.63 & -202.87 \\
\hline & 245.63 & -188.87 & & 235.63 & -202.87 \\
\hline point B & 249.63 & -188.87 & & 240.63 & -202.87 \\
\hline \multirow{14}{*}{ side $B C$} & 249.63 & -183.87 & & 245.63 & -202.87 \\
\hline & 249.63 & -178.87 & & 249.63 & -202.87 \\
\hline & 249.63 & -173.87 & point $B_{2}$ & 263.63 & -202.87 \\
\hline & 249.63 & -168.87 & \multirow{11}{*}{ side $B_{2} C_{2}$} & 263.63 & -188.87 \\
\hline & 249.63 & -163.87 & & 263.63 & -183.87 \\
\hline & 249.63 & -158.87 & & 263.63 & -178.87 \\
\hline & 249.63 & -153.87 & & 263.63 & -173.87 \\
\hline & 249.63 & -148.87 & & 263.63 & -168.87 \\
\hline & 249.63 & -143.87 & & 263.63 & -163.87 \\
\hline & 249.63 & -138.87 & & 263.63 & -158.87 \\
\hline & 249.63 & -133.87 & & 263.63 & -153.87 \\
\hline & 249.63 & -128.87 & & 263.63 & -148.87 \\
\hline & 249.63 & -123.87 & & 263.63 & -143.87 \\
\hline & 249.63 & -118.87 & & 263.63 & -138.87 \\
\hline
\end{tabular}




\begin{tabular}{|c|c|c|c|c|c|}
\hline \multirow{18}{*}{ side $B C$} & 249.63 & -113.87 & \multirow{23}{*}{ side $B_{2} C_{2}$} & 263.63 & -133.87 \\
\hline & 249.63 & -108.87 & & 263.63 & -128.87 \\
\hline & 249.63 & -103.87 & & 263.63 & -123.87 \\
\hline & 249.63 & -98.87 & & 263.63 & -118.87 \\
\hline & 249.63 & -93.87 & & 263.63 & -113.87 \\
\hline & 249.63 & -88.87 & & 263.63 & -108.87 \\
\hline & 249.63 & -83.87 & & 263.63 & -103.87 \\
\hline & 249.63 & -78.87 & & 263.63 & -98.87 \\
\hline & 249.63 & -73.87 & & 263.63 & -93.87 \\
\hline & 249.63 & -68.87 & & 263.63 & -88.87 \\
\hline & 249.63 & -63.87 & & 263.63 & -83.87 \\
\hline & 249.63 & -58.87 & & 263.63 & -78.87 \\
\hline & 249.63 & -53.87 & & 263.63 & -73.87 \\
\hline & 249.63 & -48.87 & & 263.63 & -68.87 \\
\hline & 249.63 & -43.87 & & 263.63 & -63.87 \\
\hline & 249.63 & -38.87 & & 263.63 & -58.87 \\
\hline & 249.63 & -33.87 & & 263.63 & -53.87 \\
\hline & 249.63 & -28.87 & & 263.63 & -48.87 \\
\hline Point C & 249.63 & -22.87 & & 263.63 & -43.87 \\
\hline \multirow{11}{*}{ side CD } & 245.63 & -22.87 & & 263.63 & -38.87 \\
\hline & 240.63 & -22.87 & & 263.63 & -33.87 \\
\hline & 235.63 & -22.87 & & 263.63 & -28.87 \\
\hline & 230.63 & -22.87 & & 263.63 & -22.87 \\
\hline & 225.63 & -22.87 & point $C_{2}$ & 263.63 & -8.87 \\
\hline & 220.63 & -22.87 & \multirow{13}{*}{ side $C_{2} D_{2}$} & 249.63 & -8.87 \\
\hline & 215.63 & -22.87 & & 245.63 & -8.87 \\
\hline & 210.63 & -22.87 & & 240.63 & -8.87 \\
\hline & 205.63 & -22.87 & & 235.63 & -8.87 \\
\hline & 200.63 & -22.87 & & 230.63 & -8.87 \\
\hline & 195.63 & -22.87 & & 225.63 & -8.87 \\
\hline point D & 190.63 & -22.87 & & 220.63 & -8.87 \\
\hline \multirow{18}{*}{ side DA } & 190.63 & -28.87 & & 215.63 & -8.87 \\
\hline & 190.63 & -33.87 & & 210.63 & -8.87 \\
\hline & 190.63 & -38.87 & & 205.63 & -8.87 \\
\hline & 190.63 & -43.87 & & 200.63 & -8.87 \\
\hline & 190.63 & -48.87 & & 195.63 & -8.87 \\
\hline & 190.63 & -53.87 & & 190.63 & -8.87 \\
\hline & 190.63 & -58.87 & point $\mathrm{D}_{2}$ & 176.63 & -8.87 \\
\hline & 190.63 & -63.87 & \multirow{11}{*}{ side $D_{2} A_{2}$} & 176.63 & -22.87 \\
\hline & 190.63 & -68.87 & & 176.63 & -28.87 \\
\hline & 190.63 & -73.87 & & 176.63 & -33.87 \\
\hline & 190.63 & -78.87 & & 176.63 & -38.87 \\
\hline & 190.63 & -83.87 & & 176.63 & -43.87 \\
\hline & 190.63 & -88.87 & & 176.63 & -48.87 \\
\hline & 190.63 & -93.87 & & 176.63 & -53.87 \\
\hline & 190.63 & -98.87 & & 176.63 & -58.87 \\
\hline & 190.63 & -103.87 & & 176.63 & -63.87 \\
\hline & 190.63 & -108.87 & & 176.63 & -68.87 \\
\hline & 190.63 & -113.87 & & 176.63 & -73.87 \\
\hline
\end{tabular}




\begin{tabular}{|c|c|c|c|c|c|}
\hline \multirow{14}{*}{ side DA } & 190.63 & -118.87 & \multirow{23}{*}{ side $D_{2} A_{2}$} & 176.63 & -78.87 \\
\hline & 190.63 & -123.87 & & 176.63 & -83.87 \\
\hline & 190.63 & -128.87 & & 176.63 & -88.87 \\
\hline & 190.63 & -133.87 & & 176.63 & -93.87 \\
\hline & 190.63 & -138.87 & & 176.63 & -98.87 \\
\hline & 190.63 & -143.87 & & 176.63 & -103.87 \\
\hline & 190.63 & -148.87 & & 176.63 & -108.87 \\
\hline & 190.63 & -153.87 & & 176.63 & -113.87 \\
\hline & 190.63 & -158.87 & & 176.63 & -118.87 \\
\hline & 190.63 & -163.87 & & 176.63 & -123.87 \\
\hline & 190.63 & -168.87 & & 176.63 & -128.87 \\
\hline & 190.63 & -173.87 & & 176.63 & -133.87 \\
\hline & 190.63 & -178.87 & & 176.63 & -138.87 \\
\hline & 190.63 & -183.87 & & 176.63 & -143.87 \\
\hline point $A$ & 190.63 & -188.87 & & 176.63 & -148.87 \\
\hline & & & & 176.63 & -153.87 \\
\hline & & & & 176.63 & -158.87 \\
\hline & & & & 176.63 & -163.87 \\
\hline Starting point $(\mathrm{Z})$ & 156.72 & -204.02 & & 176.63 & -168.87 \\
\hline End point (C) & 256.72 & -4.02 & & 176.63 & -173.87 \\
\hline & & & & 176.63 & -178.87 \\
\hline & & & & 176.63 & -183.87 \\
\hline & & & & 176.63 & -188.87 \\
\hline & & & side $A_{2}$ & 176.63 & -202.87 \\
\hline
\end{tabular}

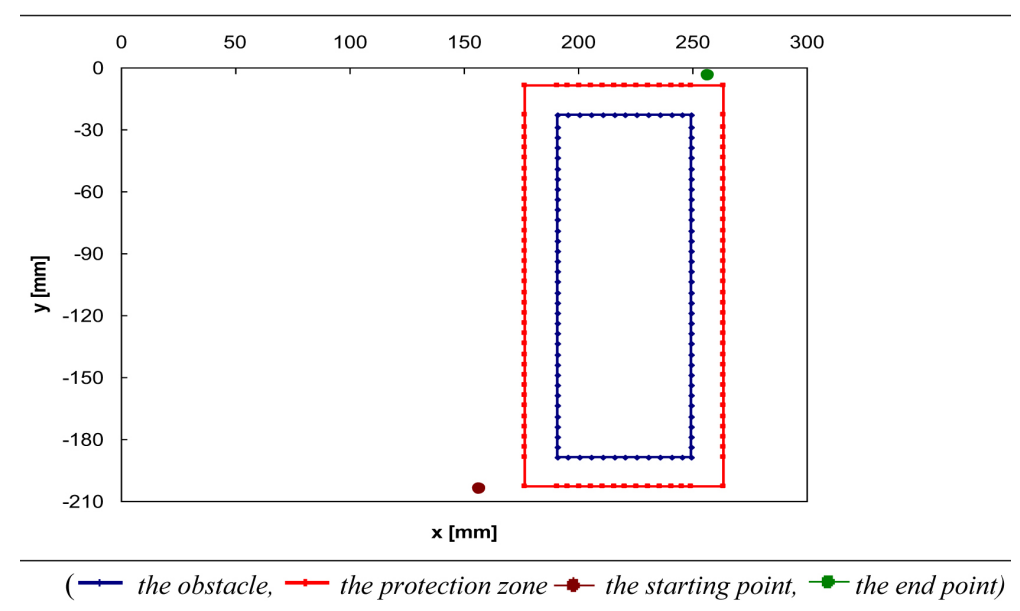

Figure 3. Graphic image of the obstacle placement together with the protection zone in the xy plane and the protection zone

are used for the coordinate points calculation employed in programming the robot trajectory.

\section{Configuration field evaluation}

In this step, the evaluation of the both fields will be provided in the following sequence:

1. All empty (non-collision) configurations form the configuration field.
2. The starting position has 0 value.

3. All neighbouring configurations with the starting configuration will be given the value of 1 .

4. All other non-valued configurations neighbouring with those with value 1 will be given the value of 2 .

5. The configurations evaluations are run till the final configuration is reached. 


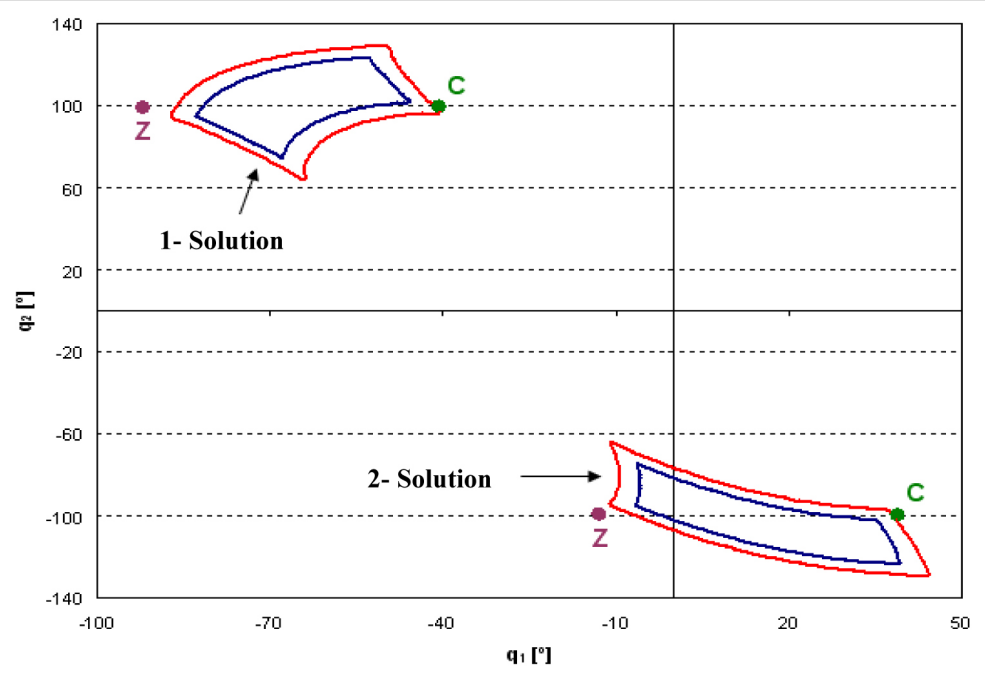

Figure 4 Image of the obstacle and the protection zone in the configuration field

(The blue line - the obstacle, The red line - the protection zone, $\mathrm{Z}$ - the starting point, $\mathrm{C}$ - the end point)

In this way, the valued configuration fields which are suitable for the search of the trajectory avoiding the obstacle will be acquired (see Figures 7 and 8).

\section{THE NON-COLLISION TRAJECTORY IDENTIFICATION FOR THE ROBOT AVOIDING THE OBSTACLE}

The aim of this step is to identify one or more paths that are non-colliding for the robot; meaning they avoid the obstacle. Identification is done in a different direction than the configuration field evaluation in the following steps:

1. From the configurations adjacent to the end configuration, the one with the lowest value is selected.

2. From the configurations adjacent to this configuration, only the one with the lowest value is selected again.

The configuration selected carried out in this way till the starting configuration is reached.

In this case, we were searching for the two path types - the closest one to the obstacle and the closest path to the obstacle with the lowest

Table 2 Increases in the rotations $\mathrm{q}_{1}$ and $\mathrm{q}_{2}$ for the first and second solution

\begin{tabular}{|c|c|c|}
\hline & $\Delta \mathrm{q}_{1}\left[^{\circ}\right]$ & $\Delta \mathrm{q}_{2}\left[^{\circ}\right]$ \\
\hline 1st solution & 1.33 & 4 \\
\hline 2nd solution & 1.9 & 3.33 \\
\hline
\end{tabular}

number of the cranks in the used configuration field. While searching the paths in the configuration field, it is important to take into consideration that the distance between the two adjacent configurations is constant also in terms of the values of the rotations $\mathrm{q}_{1}$ or $\mathrm{q}_{2}$. In the case of programming the coordinates of $\mathrm{x}$ and $\mathrm{y}$, it is impossible to talk about the constant distance of the adjacent configurations because for their calculations, the cosine and sinusoidal formulas are applied for the sum of the rotations. This fact causes irregular changes in the coordinates $\mathrm{x}$ and $\mathrm{y}$ values when changing the values of the rotations $\mathrm{q}_{1}$ and $\mathrm{q}_{2}$ by the constant figure. This is the reason to identify two different path types for each solution which are later compared according to their length with the aim to find the shortest one.

As we can see in Figure 7, the numeric value of the target point has the same value 40 after the field evaluation around the obstacle from above and under. That is the reason why 4 trajectories were found for the $1^{\text {st }}$ solution (two closest to the obstacles and two closest to the obstacles with the lowest number of the cranks). Figure 8 shows that in the second solution, the maximum value of the numeration the target point 37 is reached while evaluating the field around the obstacle. Therefore, 2 trajectories were found for the second solution - one is the closest one to the obstacle and the second is the closest one to the obstacle with the lowest number of the cranks. All the identified trajectories (6 together) are shown in Figures 9 to 12 (the trajectories are shown in red or blue double line). 


\section{Transformation of the rotation values of $q_{1}$ and $q_{2}$ to $x$ and $y$ coordinates}

The configuration field is formed as the dependence of the rotations $\mathrm{q}_{2}$ from $\mathrm{q}_{1}$; so the individual configurations (points, the configuration nodes) are defined by these two parameters. In the case of programming, it is better to know the $\mathrm{x}$ and $\mathrm{y}$ coordinates of the points because it is easier to load them to the robot Central Processing Unit. In order to do this, it is necessary for the each point of the identified trajectories in the configuration field to execute the transformation (recalculation) of the rotation $\mathrm{q}_{1}$ and $\mathrm{q}_{2}$ values to the values of the coordinates $\mathrm{x}$ and $\mathrm{y}$. the recalculation of the rotations to the coordinates of the point on the plane xy will be done using the formulas (5) and (6) that are derived from the final transformation matrix $\mathrm{T}_{\mathrm{E}}$ (see Figure 13). The input data are the rotations $\mathrm{q}_{1}$ and $\mathrm{q}_{2}$ and the length values of the robot arms $\left(a_{1}=225 \mathrm{~mm}, \mathrm{a}_{2}=166.1 \mathrm{~mm}\right)$.

After the calculation of the point coordinates $\mathrm{x}, \mathrm{y}$ of the found trajectories, it is possible to display those trajectories together with the avoiding obstacle and its protection zone in the xy plane. Figures 15 and 16 show the trajectories stemming from Figure 9 and Figure 10 (the identified trajectories closest to the obstacle and the trajectory closest to the obstacle with the lowest number of the cranks for the $1^{\text {st }}$ solution of the obstacle position in the configuration field).

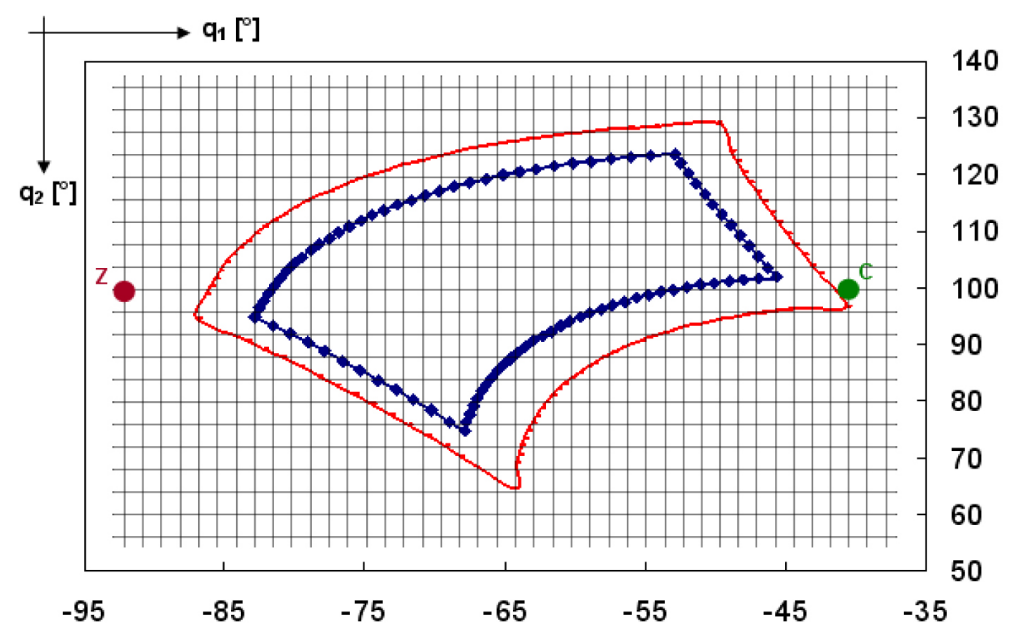

Figure 5 Configuration field together with the displayed configuration network for the $1^{\text {st }}$ solution for the obstacle and its protection zone position in the field:

(The blue line - the obstacle, The red line - the protection zone, $\mathrm{Z}$ - the starting point, $\mathrm{C}$ - the end point)

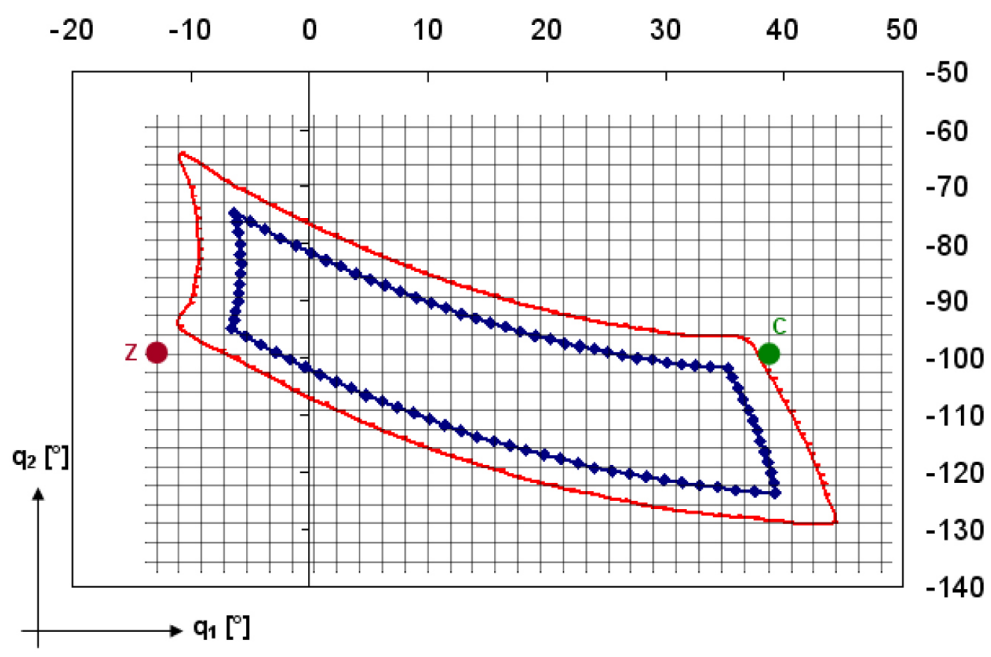

Figure 6 Configuration field together with the displayed configuration network for the $2^{\text {nd }}$ solution for the obstacle and its protection zone position in the field:

(The blue line - the obstacle, The red line - the protection zone, $\mathrm{Z}$ - the starting point, $\mathrm{C}$ - the end point) 


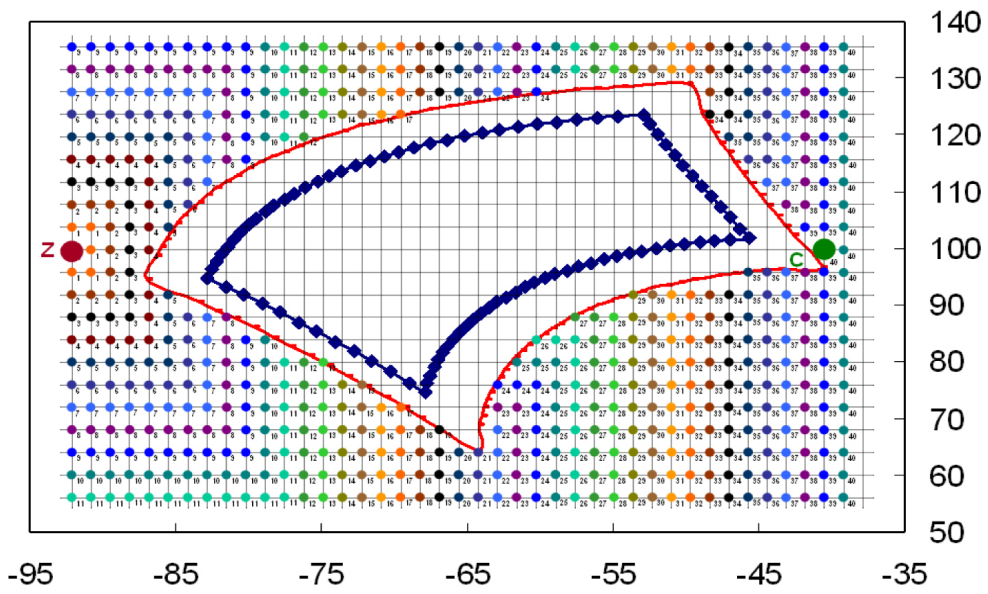

Figure 7 The evaluated configuration field for the $1^{\text {st }}$ solution for the obstacle position

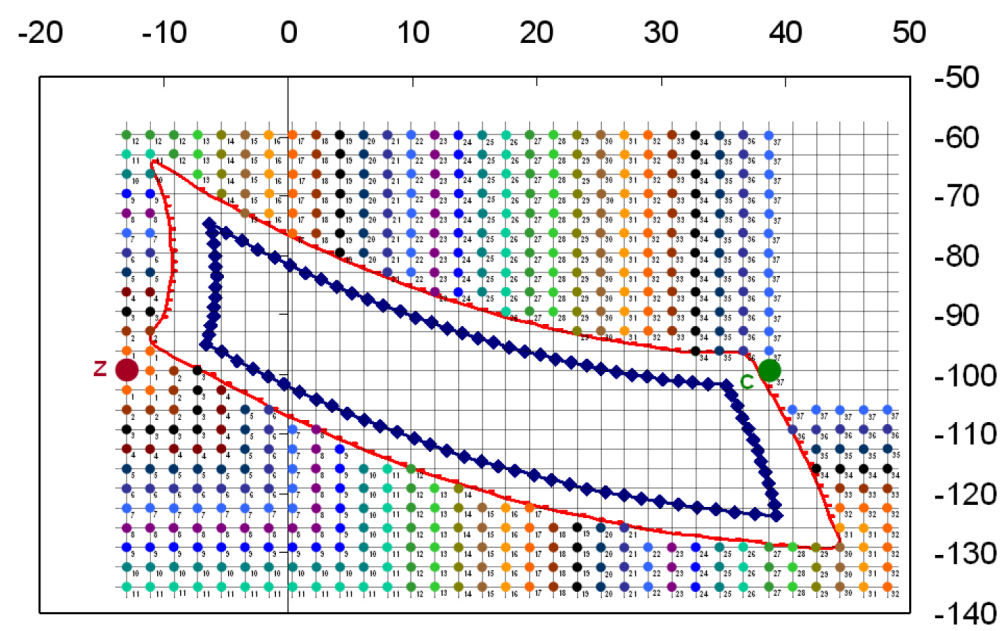

Figure 8 The evaluated configuration field for the $2^{\text {nd }}$ solution for the obstacle position

Figures 17 and 18 show the identified trajectories for the $2^{\text {nd }}$ solution of the obstacle position in the configuration field stemming from figures 11 and 12 (the closest trajectory to the obstacle and the closest trajectory with the lowest number of the cranks).

\section{DISCUSSION}

After the Lee algorithm application for the determination of the few trajectories of the robot movement around the defined obstacle and the coordinates $\mathrm{x}, \mathrm{y}$ calculation of the single points of the identified trajectories, it is possible to create the robot's manipulation motion programs. The robot used by us is Robot Yamaha YK400K and is programmed in the on-line regime directly by manual controlling unit.

There are 6 programs for the controlling of the robot manipulating movement -1 for each of the trajectory around the obstacle. The motion programming is continuous without any interruption, so it is the continuous path of the robot movement. (continuous path - CP). While programming a robot, the following steps are taken:

1. The obstacle is placed in the robot's working area - its position as well as the position of the initial and the target motion point are known (see Table 1),

2. Loading of the coordinates' points forming the trajectory for the robot movement together with the starting and the end motion point into the robot controlling unit,

3. Creation of the robot program motion itself,

4. Verification of the obstacle avoidance programme,

5. Implementation of the created programme for the obstacle avoidance.

The trajectories are compared in relation to the economics of the manipulation cycles from the two insights. It is the length and the time of the movement. 


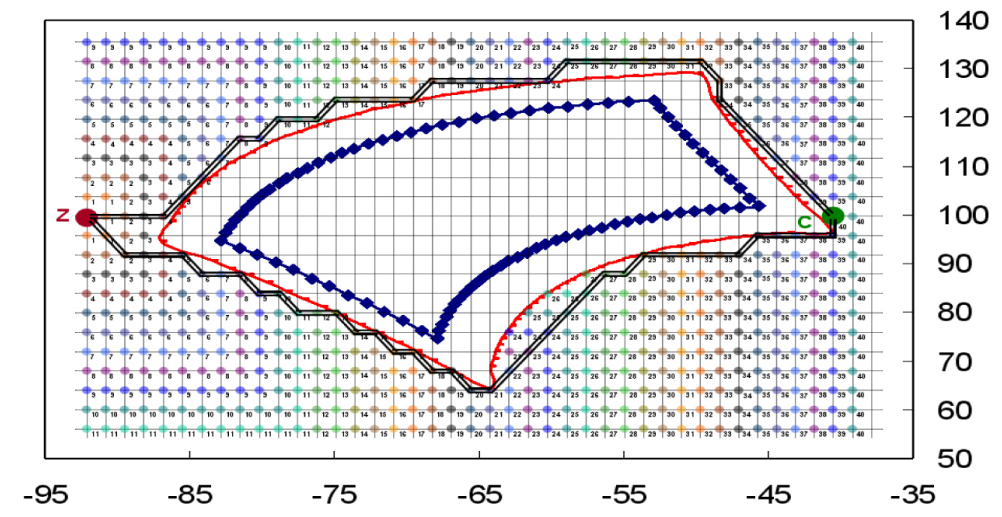

Figure 9 The identified trajectories closest to the obstacle for the $1^{\text {st }}$ solution of the obstacle position

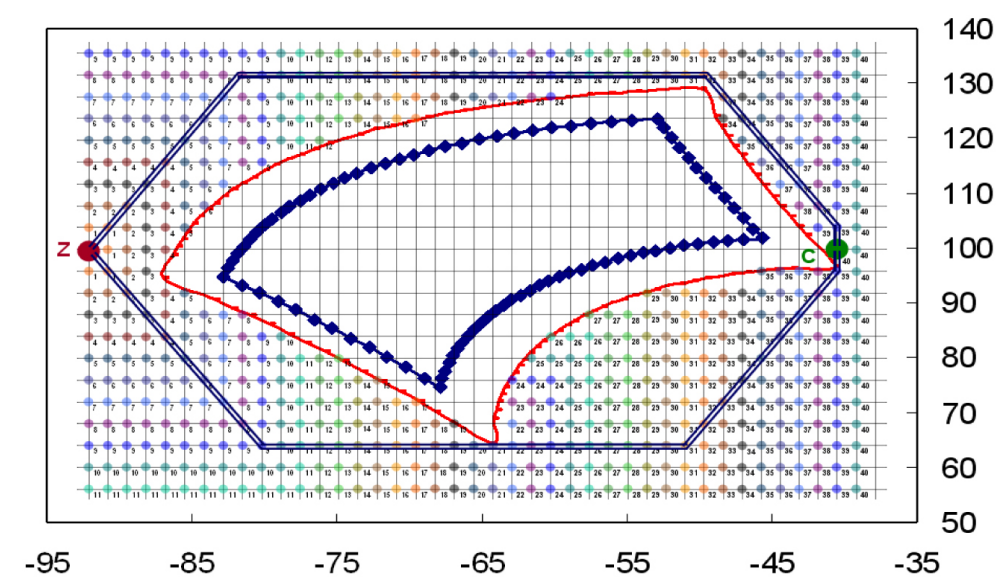

Figure 10 The identified trajectories closest to the obstacle with the lowest number of the cranks for the $1^{\text {st }}$ solution of the obstacle position

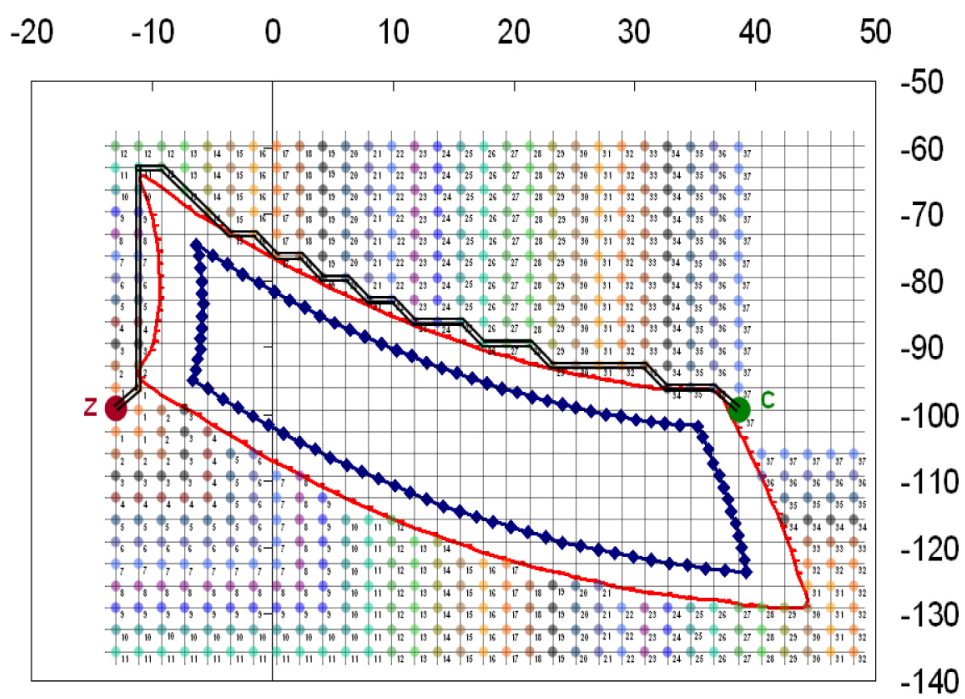

Figure 11 The identified trajectory closest to the obstacle for the $2^{\text {nd }}$ solution of the obstacle position

For the length of the identified trajectories around the obstacle, the Pythagoras' theorem is used. The calculated lengths are shown in the Table 3.

The comparing of the trajectories according to the time is allowed due to the same position of the starting and the end motion point as well as by the compliance with the executing and the number of the ongoing commands in all six programmes for the robot motion. The measured values are defined in the Table 4. 


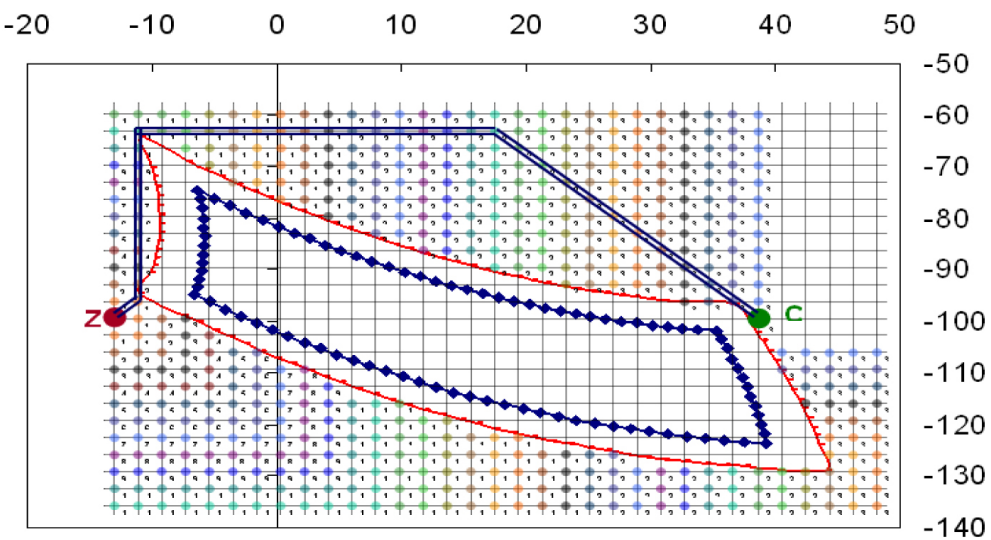

Figure 12 The identified trajectory closest to the obstacle with the lowest number of the cranks for the $2^{\text {nd }}$ solution of the obstacle position

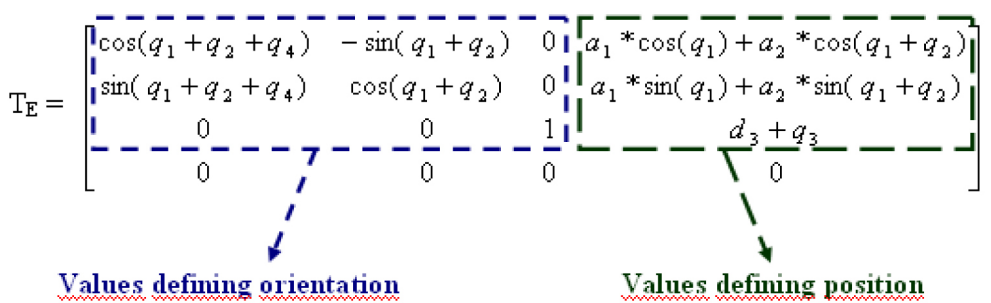

Figure 13 The final transformation matrix $T_{E}$

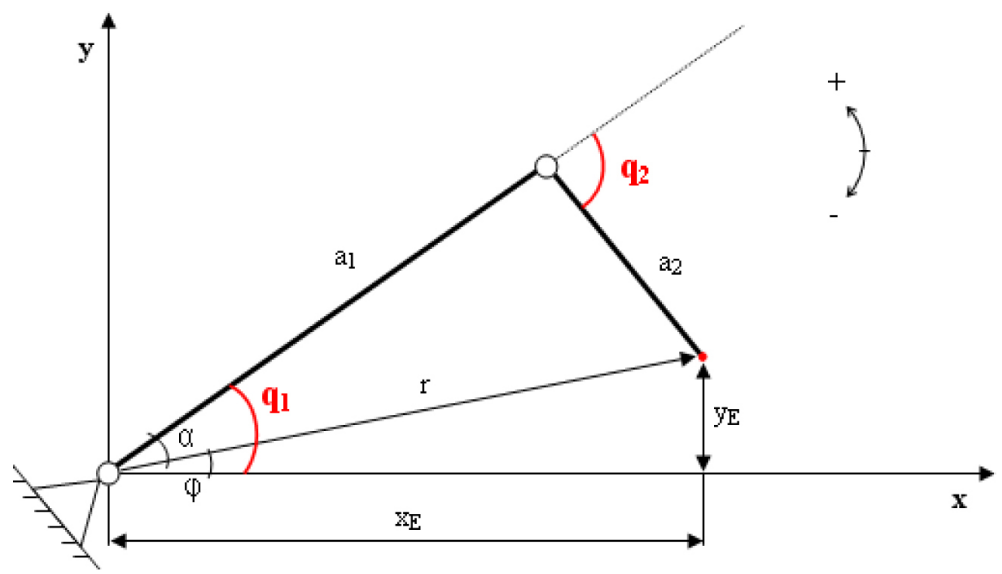

Figure 14 The image of the robot arm SCARA in the xy plane for the $\mathrm{q}_{1}$ and $\mathrm{q}_{2}$ parameter calculation

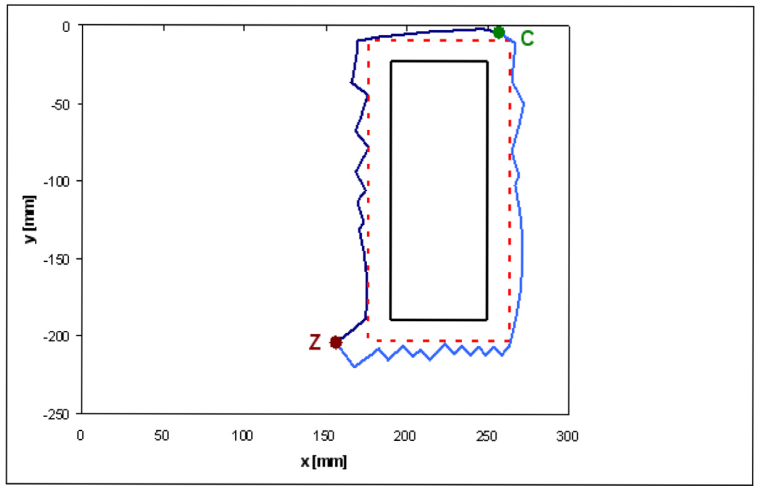

- Road number 1 , - the obstacle, - - the protection zone, - Road number 2

Figure 15 Display of the of the identified trajectories closest to the obstacle for the $1^{\text {st }}$ solution of the obstacle position in the configuration field in the xy plane 


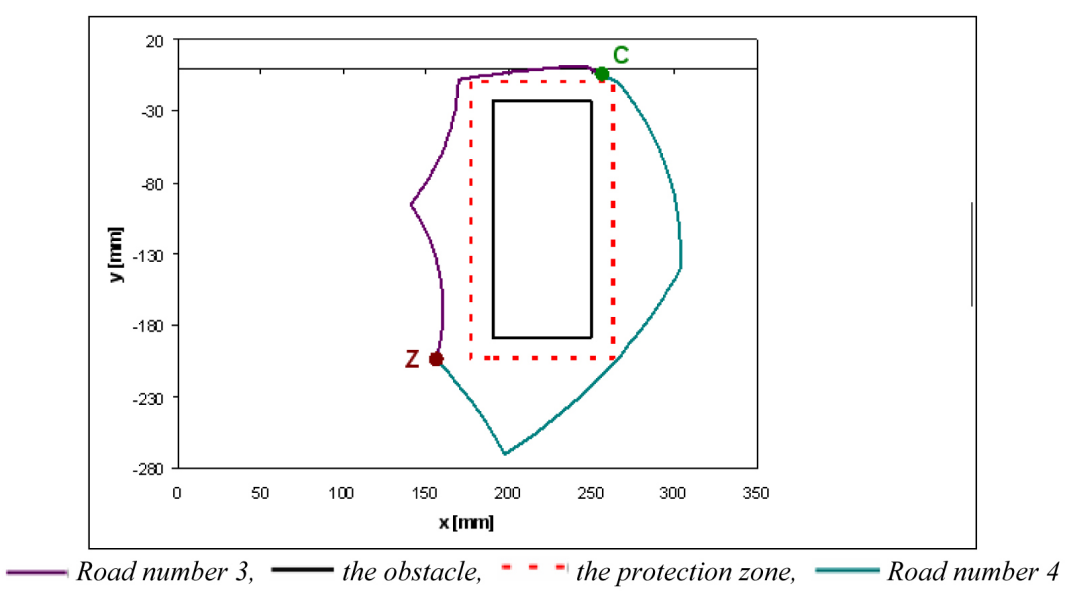

Figure 16 Display of the identified trajectories closest to the obstacle with the lowest number of the cranks for the $1^{\text {st }}$ solution of the obstacle position in the configuration field

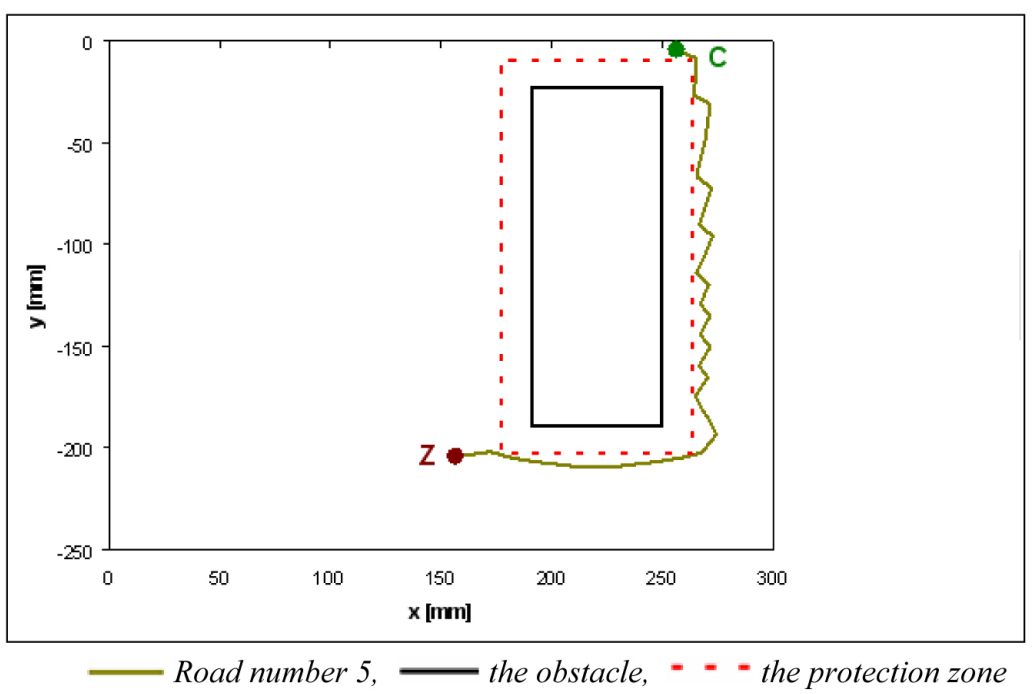

Figure 17 Display of the of the identified trajectories closest to the obstacle for the $2^{\text {nd }}$ solution of the obstacle position in the configuration field in the xy plane

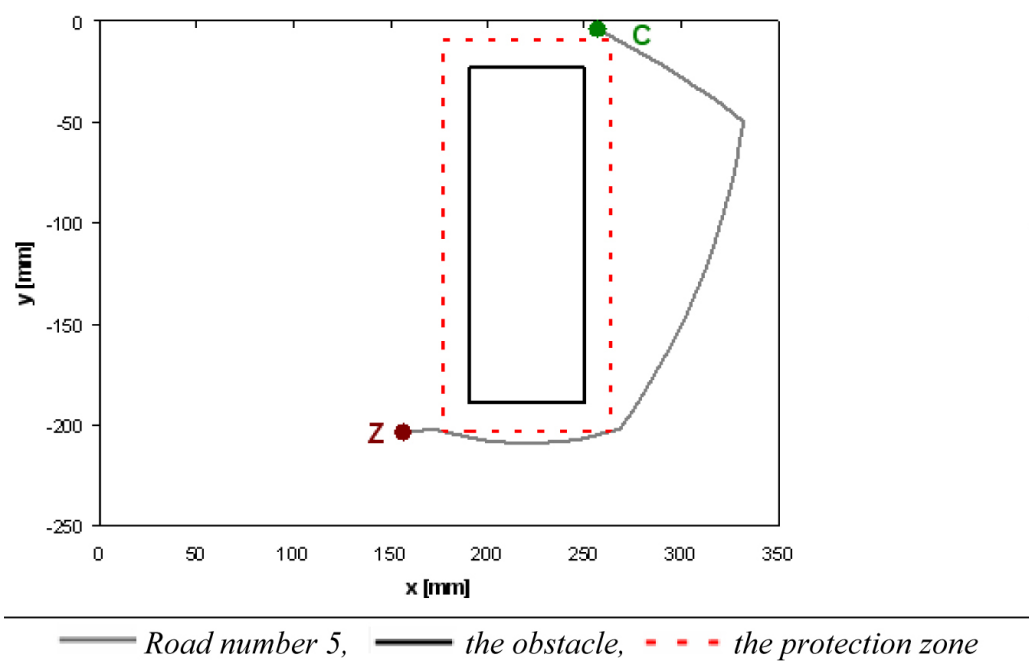

Figure 18 Display of the identified trajectory closest to the obstacle with the lowest number of the cranks for the $2^{\text {nd }}$ solution of the obstacle position in the configuration field in the xy plane. 
Table 3 The lengths of the identified trajectories for the robot obstacle avoidance

\begin{tabular}{|c|c|c|c|c|c|c|}
\hline & Trajectory No. 1 & Trajectory No. 2 & Trajectory No. 3 & Trajectory No. 4 & Trajectory No. 5 & Trajectory No. 6 \\
\hline $\begin{array}{c}\text { Leght of the } \\
\text { trajectory [mm] }\end{array}$ & 306.24 & 372.96 & $\mathbf{2 9 5 . 0 4}$ & 396.29 & 344.67 & 367.59 \\
\hline
\end{tabular}

Table 4 Times of the robot motion while avoiding the obstacle

\begin{tabular}{|c|c|c|c|c|c|c|}
\hline & Trajectory No. 1 & Trajectory No. 2 & Trajectory No. 3 & Trajectory No. 4 & Trajectory No. 5 & Trajectory No. 6 \\
\hline Time [s] & 29.47 & 32.67 & 29.11 & 33.79 & 31.36 & 32.49 \\
\hline
\end{tabular}

On the basis of the acquired data on the robot movement trajectory length and on the time length of its movement, itcanbe stated that trajectory No. 3 is optimal regarding both aspects (see Figure 15).

\section{CONCLUSION}

The planning of the robot's manipulation cycles is one of the key problems in the robot technology area. It is the security, appropriateness, noncollision and accuracy solution for the projected motion trajectory. The avoidance of obstacles and thus ensuring the safe and economic motion of an industrial robot in the application environment are the programming priority. There are few methods to prevent the robot's collision or more precisely its end effector with the obstacle and to ensure simultaneous economy of the manipulation cycles.

The Lee algorithm is quite a simple solution of securing the non-collision motion of the industrial robot around the obstacle which position in the working area of the robot is known. It is one of the methods that combine the computerized and graphic elements to specify the non-collision motion trajectory of the industrial robot. It suitably combines the kinematic robot structure and the transformation matrices calculation with the graphic search for the trajectory avoiding the obstacle in the robot configuration field. Performing all the necessary calculation, creating the configuration field, evaluating it and identifying the non-collision industrial model motion trajectory requires a lot of time for the programmer. However, the Lee algorithm still has its place among the more exact used methods of solving the problem of the obstacle avoidance in the robot working area at the workplace.

\section{Acknowledgements}

This work was supported by the Slovak Research and Development Agency under the contract No. APVV-16-0485 and VEGA 1/0376/17.

\section{REFERENCES}

1. Angeles J.: Fundamentals of Robotic Mechanical Systems (Theory, Methods, and Algorithms) Montreal. Springer, 2007.

2. Ceng J., Krämer S.: OOP-Project Maze Router with Lee Algorithm. Aachen. Institute for Integrated Signal Processing Systems, 2007.

3. Daneshjo N., Králik M., Majerník M., Dudáš Pajerská E., Naščáková J.: Non-collision trajectories of service industrial robots. Advances in Engineering Software, 124 (2018), 90-96.

4. Daneshjo N., Korba P., Vargová R., Tahzib B. Application of 3D Modeling and Simulation Using Modular Components. Applied Mechanics and Materials, 389 (2013), 957-962.

5. Gálisová L., Knežo D.: Macroscopic ground-state degeneracy and magnetocaloric effect in the exactly solvable spin-12 Ising-Heisenberg doubletetrahedral chain. Physics Letters A. 39 (2018), 2839-2845.

6. Knežo, D., Andrejiová, M., Kimáková, Z., Radchenko, S.: Determining of the optimal device lifetime using mathematical renewal models. TEM Journal. 2 (2016), 121-125.

7. Manová E., Čulková K., Lukáč J., Simonidesová J., Kudlová J.: Position of the Chosen Industrial Companies in Connection to the Mining. Acta Montanistica Slovaca. Košice. Technical University of Košice, 23(2018), 132-140.

8. Palko A., Smrček J.: The use of pneumatic artificial muscles in robot construction. Industrial Robot - An International Journal. 1 (2011), 11-19.

9. Pribulová A., Futáš P., Petrík J., Pokusová M., Brzezinski, M., Jakubski, J.: Comparison of cupola furnace and blast furnace slags with respect to possibilities of their utilization. Archives of Metallurgy and Materials. 4 (2018), 1865-1873.

10. Semjon J., Sukop M., Vagaš M., Jánoš R., Tuleja P., Koukolová L., Marcinko P., Juruš O., Varga J.: Comparison of the delta robot ABB IRB 360 properties after collisions. Communications - Scientific Letters of the University of Zilina. 1 (2018), 42-46. 